\title{
Increasing the Piezoactivity of Manufactured Composite Piezoceramic Transformers by Combining the Processes of Soldering and Polarization
}

\author{
Yurii Paerand* (Professor, Volodymyr Dahl East Ukrainian National University, Luhansk, Ukraine), \\ Oleh Zakhozhai (Professor, Volodymyr Dahl East Ukrainian National University, Luhansk, Ukraine)
}

\begin{abstract}
The article is devoted to solving the problem of ensuring high piezoactivity of composite piezoceramic transformers, in which the component parts are connected by soldering. The existing connection features have been analysed for the elements of composite piezoceramic transformers and the results have shown that during soldering the piezoceramic elements are exposed to thermal shock leading to depolarization of piezoceramics and deterioration of its piezoelectric properties. Diminution of temperature depolarizing effect is possible using low-temperature solders with a high tin content. However, in this case, the diffusion process of the electrode material of the piezoelectric element into the solder increases and the mechanical connection strength decreases. To eliminate these negative phenomena and solve the problem, a new method has been developed for connecting elements of composite piezoceramic transformers by soldering, where a polarizing voltage is applied to the composite piezoceramic transformer when heating and cooling, which prevents depolarization of the domain structure of piezoceramic elements. At the same time, high temperature ensures that polarization is carried out at lower values of the electric tension, and a more mobile domain structure after polarization has a lower level of coercive forces and, accordingly, a greater stability of parameters over time. Experimental verification has shown that the relative resonance gap increases by $6 \%$ with a decrease in the dispersion of this value by $12 \%$, which indicates an increase in piezoactivity and stability of the characteristics of composite piezoceramic transformers manufactured using the solution above.
\end{abstract}

Keywords - Piezoelectric devices; Piezoelectric materials; Piezoelectric polarization.

\section{INTRODUCTION}

In modern technology, piezoelectric ceramics and piezoelectric converters are widely used in the manufacturing of various electronic components and devices such as frequency-dependent components, micro-motion devices, sensors, etc. [1], [2]. The performance of such converters greatly affects the quality of the end devices. Piezoactivity is one of the major parameters for piezoelements. Therefore, the development of effective methods and means of increasing piezoactivity is an important scientific and technical problem.

Most devices use a unit being a mechanical connection of piezoceramic elements to each other, or piezoceramic elements to a metal plate [1], [2]. As a result of such a connection, a rigid structure with new properties is formed that depends on the properties and dimensions of its constituent elements, as well as the technology of their connection. This design in various sources is called composite piezoelectric transformer [3]-[5], bimorph and trimorph elements [6]-[9] or the sandwich type [10], [11] (in the English language sources - piezoelectric sandwich transformers [12]-[15]). The authors consider the term "composite piezoceramic transformer" (CPT) to more accurately reflect the design features, and it will be used below.

\section{Problem ANALYSiS AND PROBLEM StATEMENT}

The wide field of application of CPTs [6] has led to the development of a wide variety of their designs [1], [2], [6]. Figure 1 shows the most typical designs of the CPT. That design includes piezoceramic elements 1 , metal resonators 2 , and in some designs - plastic parts 3 . In Fig. 1, number 1 designates elements made of piezoceramics, number 2 - of metal and number 3 - of plastic.

The quality factor as a quantitative characteristic of the resonant properties of the CPT characterises the ability of the resonator to keep up the mechanical energy stored in it. Its value can be determined from the dependence [7]:

$$
Q_{\mathrm{M}}=\frac{f_{\mathrm{a}}^{2}}{2 \pi R_{\mathrm{r}} C_{0} f_{\mathrm{r}}\left(f_{\mathrm{a}}^{2}-f_{\mathrm{r}}^{2}\right)},
$$

where: $R_{\mathrm{r}}$ - resonator resistance on resonant frequency; $f_{\mathrm{r}}$ and $f_{\mathrm{a}}$ - resonant and anti-resonant frequency of piezoresonator; $C_{0}-$ static electric capacity of piezoceramic resonator at a frequency of $1000 \mathrm{~Hz}$.

In [7], the authors indicate that connection of two piezoelectric elements or a piezoelectric element and a metal

\footnotetext{
* Corresponding author.

E-mail: paerand@snu.edu.ua
} 
plate in one design leads to a significant change in the characteristics of the transformer. For example, the connection of a piezoelectric element with a metal resonator can significantly increase the $Q$-factor of the transformer, since the metal resonator has a much higher mechanical $Q$-factor than the piezoceramic element. According to [7], the mechanical $Q$-factor of piezoceramic materials is several hundred units, while the same characteristic for metal resonators ranges from
10 to 30 thousand [1]. As a result, the $Q$-factor of the CPT takes a value within the $Q$-factor range for the piezo-element and the metal element. Besides, the temporal and temperature stability of the CPT due to more stable characteristics of the metal resonator will also be higher than that of the piezoceramic element. In addition to the quality factor, the degree of polarization of the piezoceramics is also important, since it directly affects the piezoactivity of the CPT.
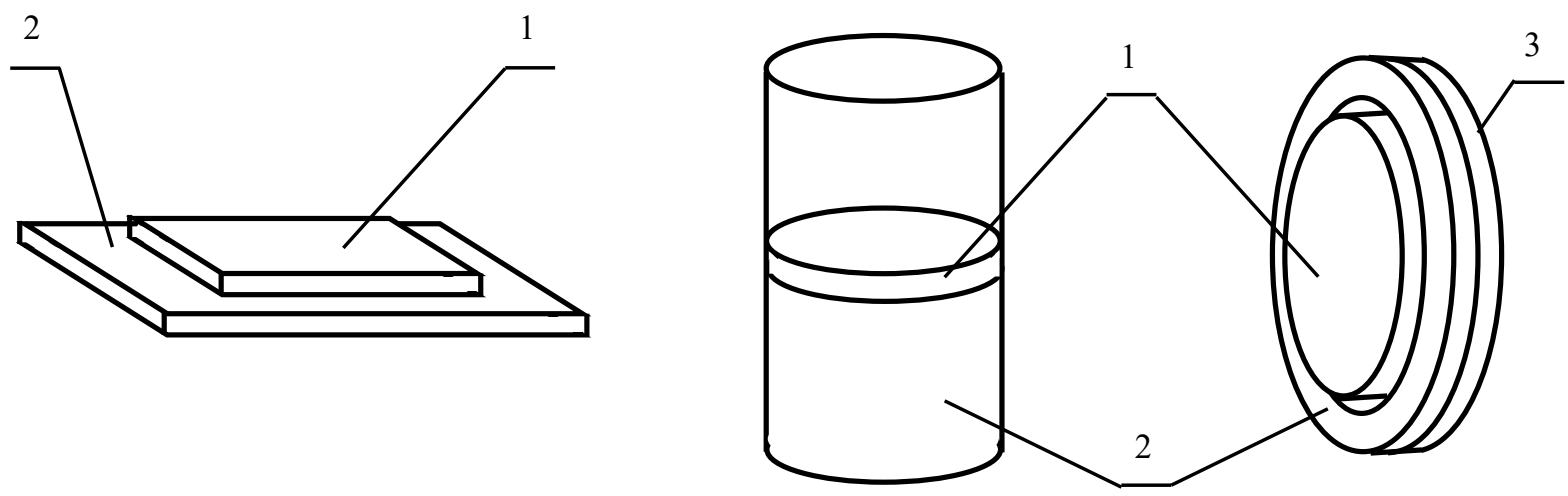

Fig. 1. Varieties of composite piezoceramic transformer structures.

Piezoactivity determines the ability of a piezoceramic element to reveal piezoelectric properties to one degree or another with a direct or reverse piezoelectric effect. For a quantitative assessment of piezoactivity, the electromechanical coupling coefficient is used, which characterises the ability of a piezoelectric element to convert energy from electrical to mechanical and vice versa. Its value is calculated by the formula [7]:

$$
K_{\mathrm{p}}=\sqrt{\frac{\eta^{2}-\sigma^{2}}{2(1+\sigma)}\left(1-\frac{f_{\mathrm{r}}^{2}}{f_{\mathrm{a}}^{2}}\right)},
$$

where: $\eta$ - the least positive root of the frequency equation; $\sigma$ - Poisson's ratio; $f_{\mathrm{r}}$ - resonant frequency, Hz; $f_{\mathrm{a}}-$ antiresonant frequency, $\mathrm{Hz}$.

Existing technologies for CPT manufacture suggest two alternative approaches to connecting the piezoelectric elements and a metal plate - by soldering or gluing. It should be noted that the characteristics of CPTs largely depend on the technology and properties of the connection. Thus, the properties and thickness of the bonding material (glue or solder) have a significant effect on the characteristics of the CPT [7].

Manufacturing experience for small-sized transformers ( $10 \mathrm{~mm}$ and less) has shown that the use of adhesive joints is impractical for two reasons. The first reason is impossibility to provide sufficient thickness repeatability and uniformity of the applied adhesive layer, leading to internal mechanical stresses that worsen the oscillatory characteristics of the CPT. The second reason is that the layer of polymerized glue has a relatively low quality factor resulting in the whole quality factor decrease of the CPT mechanical system of the connected elements.
In contrast to the glued joint, CPT manufacture by means of soldering allows providing the controlled thickness of the soldered joint, as well as a higher quality factor comparing to the adhesive one. However, the use of soldering along with the indicated advantages reveals a significant problem associated with thermal shock, which affects the degree of polarization and, as a consequence, the piezoactivity of CPT.

Growing temperature during soldering leads to depolarization of piezoelectric ceramics and decreases its piezoactivity. For this reason, to reduce thermal shock, piezoelectric elements soldering to a metal resonator requires limitation of temperature and duration of the soldering process. In this case, the use of low-temperature tin-containing solders is important. However, a high concentration of tin in the solder contributes to a higher degree of diffusion of the piezoelectric element electrode material (usually silver is used as the electrode material) into the solder. As a result of this process, the connection strength between the electrode and the piezoelectric element decreases, which leads to a diminution in the electrophysical parameters of the CPT. To reduce the negative effect of the electrode diffusion, silver is put into solder as a component leading to an increase in soldering temperatures and an increase in the depolarizing effect on the CPT manufactured.

Thus, on the basis of the analysis performed we can single out an important scientific and technical problem of ensuring high piezoactivity and quality of the CPT connection, which is in the following contradiction:

- to ensure a high-quality, high-Q connection of the composite elements of the piezoelectric transformer and to reduce the degree of contact diffusion, silver-containing solders should be used;

- usage of silver-containing solder requires high heating soldering temperatures, which increase the thermal shock 
towards piezoelectric ceramics and enhance the process of its depolarization resulting in a decrease in the CPT piezoactivity.

Thus, the characteristics of the connection quality and the CPT piezoactivity are competing and require the development of new methods and means to simultaneously ensure a highquality connection of the CPT components and high piezoactivity.

\section{AnAlysis of Possible Solutions to the Problem}

In [16], [17] the authors note that the soldering temperature is limited by the Curie temperature of the piezomaterial. Usually they are limited to an average temperature between $0{ }^{\circ} \mathrm{C}$ and the Curie temperature of the piezoelectric material. Considering that most piezomaterials have a Curie temperature of about $300^{\circ} \mathrm{C}$ soldering temperature should not exceed $1500{ }^{\circ} \mathrm{C}$. This means that the melting point of the solder must be $1200{ }^{\circ} \mathrm{C}$ and below. Such requirements complicate the manufacturing technology and reduce the operating range of the CPT. Besides, this approach does not exclude negative aspects associated with the coating contact diffusion of piezoelectric ceramics into the molten solder, but only reduces them.

Another approach is proposed by Siemens AG [18]. Its peculiarity is that the polarization of piezoelectric ceramics occurs after soldering it to a metal resonator, i.e., directly in the transformer. Thus, the restrictions on the melting temperature of the used solders and heating modes during soldering considering preventing depolarization are taken off. However, it should be noted that in this case the polarization process flows under difficult conditions. On the one hand, to increase the efficiency of polarization it is necessary to increase the temperature, the electric field intensity, and the exposure duration. On the other hand, during polarization desoldering of the piezoelectric element and the metal resonator must not occur as well as electrical breakdown, and a connection strength decrease of the transformer components.

Also, it worth mentioning that polarization efficiency depends not only on the level of the electric field intensity and the duration of exposure, but also on the temperature of the piezoceramic element. Moreover, all these parameters are interdependent and affect each other.

In [19], [20] it is indicated that with a temperature increase, the mobility of the domain structure of a piezoelectric material increases, and the coercive force decreases providing more efficient polarization at smaller electric field strength. However, in this case, there is also a negative point - as the temperature of the piezoelectric element grows, its electrical conductivity increases as well and consequently the voltage breakdown decreases. The same process is observed in strong electric fields. Under the action of an electric field with a high strength, some of the covalent bonds are destroyed and additional free carriers of electric charge appear in the structure of the piezoelectric material resulting in the leakage current increase [20]. Such a process can be of avalanche-like behaviour and lead to a breakdown of the piezoelectric element.
Electrical breakdown is also possible along the surface of the piezoelectric element. In this case, polarization is provided in a special liquid medium with a high breakdown voltage, which allows using increased electric field strengths, preventing the breakdown of the piezoelectric element along the surface. However, this technology also significantly complicates the process of manufacturing the CPT.

At a certain crystal temperature, there is some electric field strength at which an electrical breakdown starts. With the crystal temperature increase, the value of the maximum permissible electrical intensity decreases. This is explained by the fact that when temperature increases the internal energy of the crystal also increases and facilitates the process of destruction of covalent bonds [19]. The dependence of the electric breakdown strength $\left(E_{\mathrm{br}}\right)$ on the temperature of the piezoelectric element $(T)$ is described by an expression [20]:

$$
\lg E_{\mathrm{br}}=\frac{B}{T}+D
$$

where: $B$ - an index depending on piezoelectric element overall dimensions; $D$ - a constant independent of temperature; $T-$ a piezoelectric element temperature.

The polarization of piezoelectric elements for manufactured CPTs, despite the danger of electrical breakdown, is considered by the authors to be the most promising for the main reasons below:

1) the increased temperature of the piezoelectric material provides efficient polarization at smaller electric field strengths greatly simplifying the manufacturing technology for CPT;

2) higher domain structure mobility of the piezoelectric material and, as a result, lower coercive forces simplify and accelerate the process of preferential orientation of ferroelectric domains;

3 ) the predominant orientation of the domains at low coercive forces significantly reduce the aging process dynamics in CPT piezoelectric elements ensuring greater stability of their characteristics over time.

Based on the foregoing, the solution to the above-mentioned problem is ensuring the maximum polarization degree of the CPT piezoelectric elements, which increases their piezoactivity. At the same time, the quality of their constituent part connection remains critical.

\section{SOLUTION OF THE TASK}

Considering the fact that the process of CPT soldering is associated with necessary heating of the piezoelectric element and the temperature has a depolarizing effect, increasing the mobility of the domain structure, it is proposed to combine the process of soldering and polarization. In this case, the heating of the SPP will be provided under the constant influence of the polarizing voltage, which will ensure the preferential orientation of the mobile domains in the direction of the polarization vector.

According to the proposed method, a constant electric voltage is applied to the piezoelectric element during heating. 
The DC voltage is switched off after cooling the CPT to a temperature that ensures keeping up a residual polarization.

Figure 2 shows a scheme of a device that implements the proposed approach. The device contains a block for setting modes 1 , which determines the sequence and magnitude of the parameters of the technological process from a source of polarizing voltage 2 and a heating source by a resistance 3 . From source 3 through the power electrodes 4 and metal resonator 5, a current pulse is supplied, which heats it to the required temperature within a set time. From source 2 through clamps 6 , a polarizing voltage of the corresponding polarity is supplied to the piezoceramic elements 7 .

The process of soldering piezoceramic elements is carried out as follows. A tin-bismuth coating is preliminarily applied to the surface of the metal resonator by electroplating. Before soldering, piezoceramic elements and a metal resonator are assembled and fixed in the device using clamps 6 . Heating is provided by passing current through the power electrodes 4 and the metal resonator from electric current source 3 . At the same time, a polarizing voltage of the corresponding polarity is supplied to the piezoceramic elements 7 from source 2 through the clamps 6 and the power electrodes 4 . In this case, the block for setting modes 1 must provide both a high-quality mechanical connection of the CPT elements, and its required piezoactivity during further operation. The latter can be obtained by ensuring an effective process of polarization of piezoceramic elements both during heating and during their cooling.

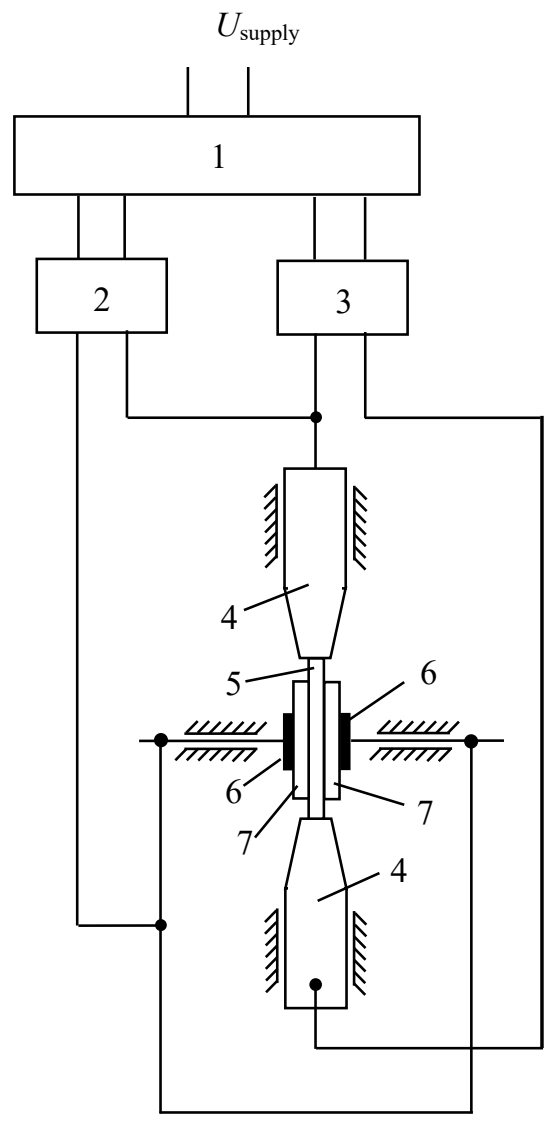

Fig. 2. Scheme of a device that implements the proposed solution.
Figure 3 shows a simplified diagram of the parameters of the CPT manufacturing process, especially the diagrams of the heating current $I$ and the polarization voltage $U$, as well as the process of changing the temperature $T$ of the CPT elements.

In the process of heating, the change in the temperature of the CPT elements in time $t$ can be represented as follows:

$$
T=T_{0}+\Delta T \cdot\left(1-e^{-t / \tau_{1}}\right),
$$

where: $T$ - the current temperature value of the CPT elements; $T_{0}$ - ambient temperature; $\Delta T=T_{\infty}-T_{0}$ - a steady-state value of overheating for heated elements (heating intensity); $\tau_{1}-\mathrm{a}$ heating time constant of a metal resonator.

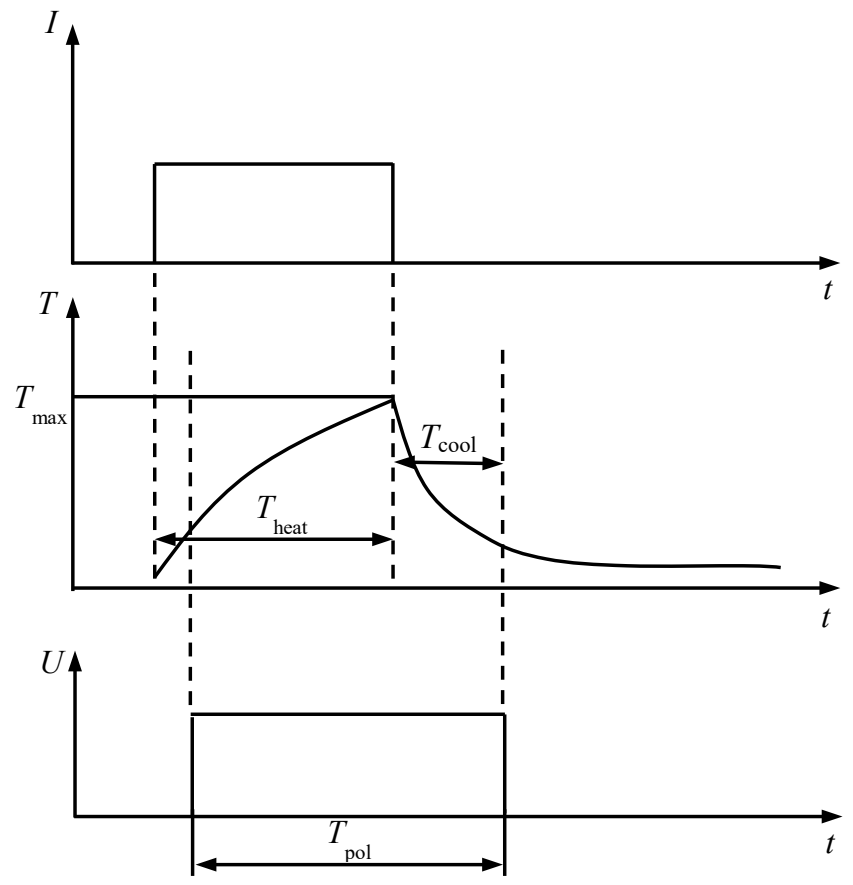

Fig. 3. Diagram of parameter change in the process of CPT soldering.

It should be noted that the steady-state temperature value under the condition of continuous heating $\left(t_{\text {heat }}=\infty\right)$ is determined by the formula:

$$
T_{\infty}=\Delta T+T_{0} .
$$

The cooling process is described by the expression:

$$
T=T_{\max } \cdot e^{-t / \tau_{2}}+T_{0},
$$

where: $T_{\max }-$ heat temperature of the metal resonator at the moment of switching off the heating source; $\tau_{2}-$ a cooling time constant of CPT.

Considering the short duration of the process, the temperature at the end of heating does not reach the steady-state value and is $T_{\max }$. This value depends on the ambient temperature, as well as on the heating intensity and duration.

Figures 4 and 5 show the dependences of the maximum temperature on the heating intensity and duration calculated by Eq. (4) for the ambient temperature $T_{0}=20^{\circ} \mathrm{C}$, heating time range $t_{\text {heat }}$ (Fig. 4 ) $-0.8 ; 1.0 ; 1.2 ; 1.4 ; 1.6 ; 1.8$ and $2.0 \mathrm{~s}$ and 
range of steady-state temperature values of soldered elements (i.e., at $\left.t_{\text {heat }}=\infty\right) T_{\infty}-370 ; 420 ; 470 ; 520 ; 570{ }^{\circ} \mathrm{C}$. The indicated ranges were selected on the basis of preliminary experiments for CPT samples operating on longitudinal vibrations with the

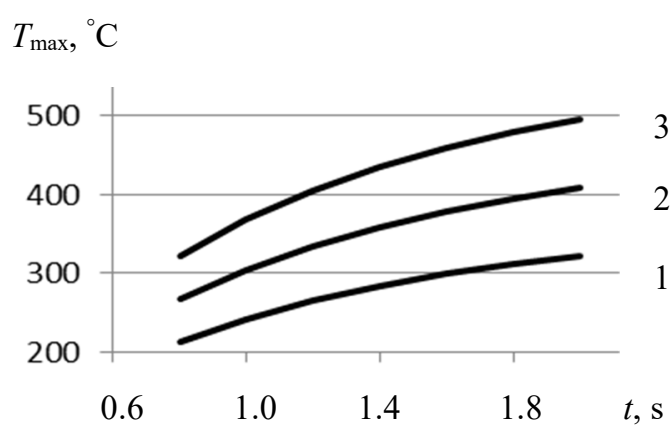

Fig. 4. Dependence of the maximum temperature $T_{\max }$ on heating time $t_{\text {heat }}$ at $T_{\infty}: 1-370{ }^{\circ} \mathrm{C} ; 2-470{ }^{\circ} \mathrm{C} ; 3-570{ }^{\circ} \mathrm{C}$

\section{CHeCKING THE EFFECTIVENESS OF THE PROPOSED SOLUTION}

To evaluate the effectiveness of the proposed solution, an experiment was carried out for groups of manufactured CPTs at $300 \mathrm{kHz}$ frequency based on ЦТС-35 ceramics. Considering (2), the electromechanical coupling coefficient depends on the design features of the piezoelectric elements, and in order to exclude additional aspects affecting the indicators, the characteristic of the relative resonance gap of the CPT was chosen as a value of piezoactivity. In fact, in relation (2) the numerator determines the difference between antiresonance and resonance. Thus, the piezoactivity assessment was made only by direct measurable parameters. The relative resonant gap of the manufactured CPT was determined as follows:

$$
\delta=\frac{f_{\mathrm{a}}-f_{\mathrm{r}}}{f_{\mathrm{r}}} .
$$

Measuring the CPT parameters was carried out according to the technique described in [13] and with the observance of the same measurement conditions. For measurements, the circuit shown in Fig. 6 was used.

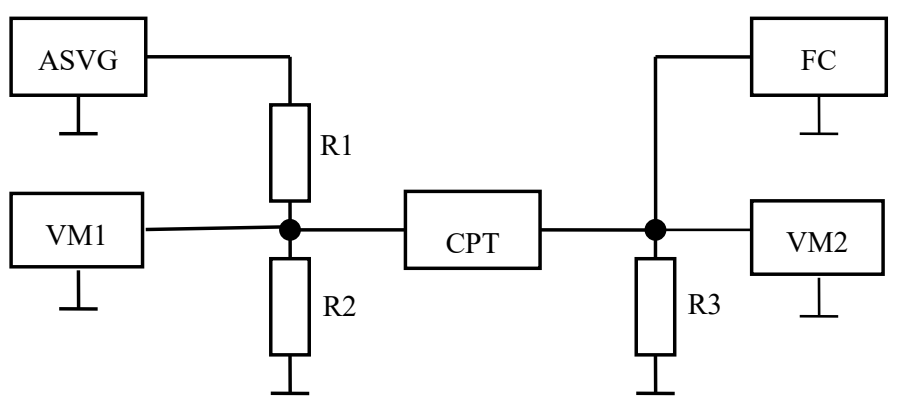

Fig. 6. Scheme for measuring CPT characteristics: ASVG - an adjustable sinusoidal voltage generator; VM1 - a voltmeter that registers the generator output voltage level; FC - a frequency counter; VM2 - a voltmeter that registers the level of the output signal. dimensions of a metal resonator made of 44HXMT alloy sized $7.85 \mathrm{~mm} \times 1.5 \mathrm{~mm} \times 0.6 \mathrm{~mm}$ and ЦТС-35 piezoelectric element sized $5.8 \mathrm{~mm} \times 1.0 \mathrm{~mm} \times 0.5 \mathrm{~mm}$.

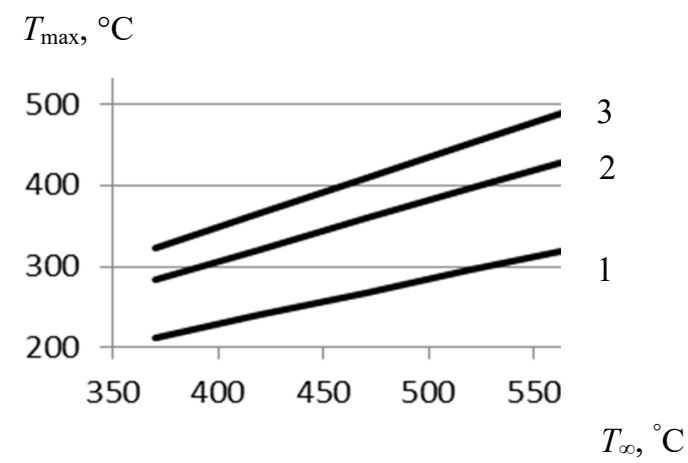

Fig. 5. Dependence of the maximum temperature $T_{\max }$ on $T_{\infty}$ at heating time $t_{\text {heat }}: 1-0.8 \mathrm{~s} ; 2-1.2 \mathrm{~s} ; 3-2.0 \mathrm{~s}$.

To ensure the same probability of a mean value of the result, the experiment was carried out for five groups of 20 CPTs in each.

\section{CONCLUSIONS AND AREAS FOR FURTHER RESEARCH}

As a result of the experiment, the average value of the relative resonance gap was determined $\bar{\Delta}_{p}=5.146 \cdot 10^{-3}$ at reproducibility variance $2.0420 \cdot 10^{-9}$. At the same time, the relative resonance gap increased by $6 \%$ with the dispersion decrease by $12 \%$ indicating the piezoactivity growth and stability of the characteristics of CPTs manufactured using the proposed solution.

The presented approach to the temporal combination of the soldering and polarization processes in performing the CPT connection of the CPT elements is aimed not only at ensuring the required mechanical strength of the connection, but also at achieving a high piezoactivity of the manufactured transformer.

Usage of combined soldering and polarization processes provides both pre-polarized and non-polarized piezoelectric element application.

For further research, the authors consider it expedient to study the modes and dynamics of heating, cooling, polarization of the CPT in order to create a dynamic model of the CPT soldering and polarization process. Such a model will enable determining rational values of technological parameters for various types and configurations of CPT to maximize their piezoactivity.

\section{REFERENCES}

[1] R. A. Johnson, Mechanical Filters in Electronics. New York: Wiley, 1983.

[2] C. H. Wong, Z. Dahari, A. Abd Manaf and M. A. Miskam, "Harvesting Raindrop Energy with Piezoelectrics: a Review," Journal of Electronic Matherials, 2014. https://doi.org/10.1007/s11664-014-3443-4

[3] Y. E. Paerand, V. A. Kulik, B. G. Chistyakov and V. A. Sergeev, "A device for soldering composite piezoceramic transducers," Patent 1145558 USSR, MKI V23K 3/00. 
[4] Y. E. Paerand and V. S. Kharitonov, "Method of manufacturing composite piezoceramic converters," Patent 1145887 USSR, MKI H03H $3 / 02$.

[5] Y. E. Paerand and V. A. Kulik, "Method of regulating the coefficient of electromechanical connection of composite converters," Patient 1477212 USSR, MKI N03N 3/02.

[6] V. M. Sharapov, M. P. Musienko and E. V. Sharapova, Piezoelectric sensors. Moscow: Technosphere, 2006.

[7] V. M. Sharapov, Eds., Piezoelectric converters. Cherkassy: ChSTU, 2004

[8] V. M. Sharapov, E. S. Polishchuk, Sensors: Reference Manual. Moscow: Technosphere, 2012.

[9] V. Nikiforov, V. Klimashin and A. Safronov, "Bimorph piezoelectric elements: actuators and sensors", Components and technologies, vol. 4, 2003, pp. 46-48.

[10] A. A. Novikov, Y. B. Shuster, D. A. Negrov and T. K. Denisova "Ultrasonic vibrating system", Patient 2465071 RF, MKI B06B 1/06

[11] S. S. Volkov and B. Y. Chernyak, Ultrasonic welding of plastics. Moscow: Chemistry, 1986.

[12] L. Shuyu, "Analysis of the sandwich piezoelectric ultrasonic transducer in coupled vibration," The Journal of the Acoustical Society of America, vol. 117, no. 2, 653, 2005. https://doi.org/10.1121/1.1849960

[13] X. Wei, Y. Yang, W. Yao and L. Zhang, "PSpice Modeling of a Sandwich Piezoelectric Ceramic Ultrasonic Transducer in Longitudinal Vibration," Sensors, vol. 17, no. 10, 2017. https://doi.org/10.3390/s17102253

[14] L. Shuyu, "Design of piezoelectric sandwich ultrasonic transducers with large cross-section," Applied Acoustics, vol. 44, no. 3, pp. 249-257, 1995. https://doi.org/10.1016/0003-682X(94)00022-N

[15] L. Shuyu and T. Hua, "Study on the sandwich piezoelectric ceramic ultrasonic transducer in thickness vibration," Smart Materials and Structures, vol. 17, no. 1, 2008. https://doi.org/10.1088/0964-1726/17/01/015034

[16] J. Van Randeraat, Piezoelectric Ceramics. Mullard, 1974.

[17] G. L. Filatov, E. F. Bayev and V. S. Tsymbalyuk, Small-sized lowfrequency mechanical filters. Moscow: Svyaz, 1974

[18] A. Gunther, H. Albsmeier and K. Traub, "Mechanical channel filters meeting CCITT specification" Proc. IEEE, vol. 67, no. 1, 1979, pp. 102 108. https://doi.org/10.1109/PROC.1979.11205

[19] E. G. Fesenko, A. Y. Danziger and O. N. Rozumovskaya, New piezoceramic materials. Rostov-on-Don: Russian State University, 1983.

[20] E. G. Fesenko Polarization of piezoceramics. Rostov-on-Don: Russian State University, 1968.

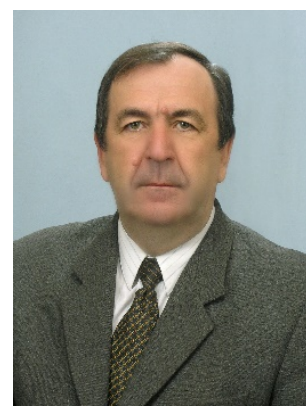

Yurii Paerand graduated from Komunarsk Mining Metallurgical Institute in 1974 with a degree in electronic apparatus. He obtained a $\mathrm{PhD}$ degree in radiotechnic and communication devices in 1986.

$\mathrm{He}$ is the Head of Electronic Apparatus Chair at Volodymyr Dahl East Ukrainian National University (Ukraine). Previous job - Head of Electronic Systems Chair at Donbass State Technical University (Ukraine), also Head of Scientific Laboratory at scientific and research institute "Phonone" (Moscow).

electronics, power electronics.

Scientific interests: piezoelectricity, high-voltage

Postal address: Tsentralnyi Ave. 59-a, Sverodonetsk, Ukraine 93400.

E-mail: paerand@snu.edu.ua

ORCID iD: https://orcid.org/0000-0002-0648-2894

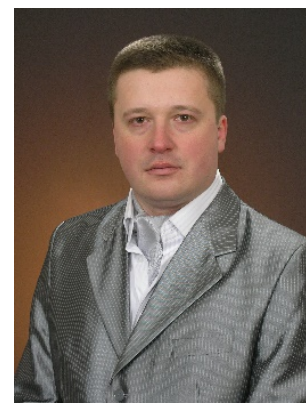

Oleh Zakhozhai graduated from Donbass State Technical University in 1999 with a degree in electronic systems. He obtained a PhD degree in automate control systems and progressive information technologies in 2004. Dr. sc. in information technologies in 2020.

$\mathrm{He}$ is a Professor of Electronic Apparatus Chair and Professor of Programming and Mathematic Chair at Volodymyr Dahl East Ukrainian National University (Ukraine). Previous job - Associate Professor of Electronic Systems Chair at Donbass State Technical University (Ukraine).

Scientific interests: information technologies, pattern recognition, information technology, electronics, microprocessor technique, IoT.

Postal address: Tsentralnyi Ave. 59-a, Sverodonetsk, Ukraine 93400.

E-mail: zoi@snu.edu.ua

ORCID iD: https://orcid.org/0000-0002-9078-3242 